\title{
Correction to: Sequent Systems for Negative Modalities
}

Ori Lahav, João Marcos and Yoni Zohar

Correction to: Log. Univers. 11 (2017), 345-382

$$
\text { https://doi.org/10.1007/s11787-017-0175-2 }
$$

In the original publication, the corresponding author was indicated incorrectly. The correct corresponding author of the article should be as mentioned below: Ori Lahav

The original article has been updated accordingly.

Ori Lahav

Max Planck Institute for Software Systems (MPI-SWS)

Kaiserslautern

Germany

e-mail: orilahav@mpi-sws.org

João Marcos

Federal University of Rio Grande do Norte

Natal

Brazil

e-mail: jmarcos@dimap.ufrn.br

Yoni Zohar

Tel Aviv University

Tel Aviv

Israel

e-mail: yoni.zohar@cs.tau.ac.il

The original article can be found online at https://doi.org/10.1007/s11787-017-0175-2. 